To cite: Magdalena Żemojtel-Piotrowska, University of Gdansk, Jarosław Piotrowski, University of Social Sciences and Humanities, Poznań Campus, John Maltby, Leicester University (2015). Agentic and Communal Narcissism and Satisfaction with Life: The Mediating Role of Psychological Entitlement and Self-esteem. International Journal of Psychology, in press.

\title{
Agentic and Communal Narcissism and Satisfaction with Life: The Mediating Role of Psychological Entitlement and Self-esteem
}

\begin{abstract}
The current study examined the mediational role of self-esteem (as an enhancement) and psychological entitlement (as a cost) in the relationship between an agentic-communal model of grandiose narcissism and satisfaction with life. Two hundred and forty-eight university undergraduate students completed measures of agentic and communal narcissism, selfesteem, psychological entitlement and satisfaction with life. The findings suggest that there is support for the usefulness of the agentic-communal model of narcissism, and, consistent with predictions in the wider literature, self-esteem and psychological entitlement mediated the relationship between agentic-communal narcissism and life satisfaction.
\end{abstract}

Key words: agentic and communal narcissism, psychological entitlement, self-esteem, satisfaction with life

Traditional considerations have placed narcissism as having a maladaptive influence on psychological well-being (Raskin \& Terry, 1988), be it via a personality disorder (Freud, 1914), a particular set of traits (Miller \& Campbell, 2008), or a culture of narcissism (Twenge \& Campbell, 2009). However, some authors have questioned the homogeneity of these reported effects of narcissism on subjective well-being (e.g. positive and negative affect, satisfaction with life; Gebauer, Sedikides, Verplanken, \& Maio, 2012; Miller \& Campbell, 2008; Rose, 2002). The positive influence of narcissism on subjective well-being is argued to 
occur via the enhancement of self-esteem (e.g. Rose, 2002; Sedikides, Rudich, Gregg, Kumashiro, \& Rusbult, 2004; Żemojtel-Piotrowska, Clinton, \& Piotrowski, 2014). Former research assumes narcissism to be either costly or beneficial for subjective well-being. However, we assume, following Paulhus' (1998) idea of the mixed blessing of narcissism, that both effects of narcissism on subjective well-being are possible. Therefore, the aim of the current paper is to answer the question about the mixed effects of grandiose narcissism (in agentic and communal form) on subjective well-being, i.e. positive via self-esteem AND negative via psychological entitlement.

Most of the former research focuses on self-esteem in explaining how narcissism is related to subjective well-being. Higher self-esteem is supposed to produce a higher selfreported level of satisfaction with life, as narcissistic individuals not only perceive themselves in a positive context but also their happiness (Sedikides et al., 2004). Moreover, the distinction between healthy (grandiose) and pathological (vulnerable) narcissism is based on the difference between the self-esteem level and stability (Miller \& Campbell, 2008; Miller, Hoffman, Gaughan, Gentile, Maples, \& Campbell, 2011). Grandiose narcissism is associated with stable and exaggerated self-esteem, vulnerable narcissism with unstable and fragile selfesteem (Miller \& Campbell, 2008). For this reason, only vulnerable narcissism is linked with negative affectivity (Miller et al., 2011) and inconsistent findings for the relationship between narcissism and subjective well-being refer only to its grandiose aspect.

However, one of the most often reported costly manifestations of narcissism is entitlement (Raskin \& Terry, 1988; Sedikides et al., 2004), which is supposed to negatively influence subjective well-being (Twenge \& Campbell, 2009). For instance, Twenge \& Campbell (2009), in their concept of the American culture of narcissism, argue that it is associated both with unrealistically high self-evaluations and expectations of others, which 
may lead to a lower level of satisfaction with one's life. Thus, psychological entitlement could be responsible for the negative effect of narcissism on subjective well-being.

Another important aspect within narcissism's conceptualization is its nature, predominantly assumed to be agentic (Sedikides et al, 2004). However, Gebauer et al. (2012) postulated that there is a distinction between agentic and communal grandiose narcissism. Communal narcissism is based on a grandiose self-view in communal domains instead of the typically reported grandiose phantasy about having agentic traits (see Gebauer et al., 2012; Sedikides et al., 2004 for reviews). However, as communal narcissists satisfy the very same narcissistic motives (i.e. grandiosity, power, self-esteem, and entitlement) as agentic narcissists do, it seems that communal narcissism is in fact another form of grandiose narcissism (see Gebauer et al., 2012). As agentic and communal narcissism are postulated as operating in a parallel manner, it is possible that beneficial and costly effects of narcissism in terms of life satisfaction could be observed for both forms.

Given the contradictory findings in regards to narcissism and satisfaction with life, we formulate two research questions to address this problem: (1) Does grandiose narcissism have mixed associations with subjective well-being, i.e. positive via self-esteem and negative via psychological entitlement? (2) Would these mixed associations be similar for its agentic and communal forms? We predict that there will be positive relationships between narcissism, psychological entitlement and self-esteem, with self-esteem mediating the relationship between narcissism and higher life satisfaction (Sedikides et al., 2004). Further, we predict, consistent with the "costly manifestation" hypothesis (Twenge \& Campbell, 2009), that psychological entitlement will mediate the relationship between narcissism and life satisfaction, leading to lower levels of the latter. Finally, congruent with the agencycommunal model of narcissism (Gebauer et al., 2012), we assume that agentic and communal narcissism operate in a similar manner. 


\section{Method}

\section{Participants}

Data were collected from a sample of 248 social science and humanities undergraduate students, from two different university populations, one in Poland $(n=137 ; 47.4 \%$ men, aged from 18 to 47 years, $M=21.56, S D=3.55)$ and one in England $(n=111 ; 26 \%$ men, aged from 18 to 30 years, $M=20.07, S D=2.61$ ). We collected data from two populations to demonstrate the cross-cultural stability of the findings.

Measures

Narcissistic Personality Inventory-13 (NPI-13, Gentile, Miller, Hoffman, Reidy, Zeichner, \& Campbell, 2013). The NPI-13 serves as a measure of agentic narcissism, with the scale demonstrating reliability and validity, and is an abbreviated version of the NPI (Raskin \& Terry, 1988). The scale comprises 13 items (e.g. "I like to look at my body"). In the current study, we used 11 items that are common to the Polish and British versions. In the English sample, participants were asked to choose between Yes and No options. In the Polish sample, participants answered on a scale from $1=$ "it's not me" to $5=$ "it's me". To establish some consistency between the two response formats, responses for the Polish sample were recoded so that scores from 1 to 3 became 0 (indicating No) and scores of 4 or 5 became 1 (indicating Yes). We then calculated overall scores for agentic narcissism. Recoding the scores from the Polish sample resulted in two effects: decreased internal consistency and decreased strength of correlations between NPI-13 and other measures (see Table 1 for details). However, the Cronbach's alphas after the recoding were comparable in both samples (see Table 1).

Communal Narcissism Inventory (Gebauer et al., 2012). This 16-item inventory serves as a measure of communal narcissism (e.g. "I am an amazing listener"). Participants answered questions on a seven-point scale from 1 = "strongly disagree" to 7 = "strongly agree". The scale has demonstrated reliability and validity (Gebauer et al., 2012). The Polish version was 
obtained by an independent back-translation procedure and a checking of the correlation of the inventory with the other scales as a part of another study (Żemojtel-Piotrowska et al., 2015).

Satisfaction with Life Scale (Diener, Emmons, Larsen, \& Griffin, 1985). This scale consists of five items, each measuring general satisfaction with one's life. Responses are scored on a five-point scale (from $1=$ "I disagree" to 5 = "I agree"). The scale has demonstrated reliability and validity (e.g. Diener et al., 1985).

Psychological Entitlement Scale (Campbell, Bonacci, Shelton, Exline, \& Bushman, 2004). This scale comprises nine items (e.g. "I deserve the best because I'm worthy of it") and serves as a measure of psychological entitlement, defined as the pervasive sense that the individual deserves more, and is entitled to more, than others. Participants responded to questions on a seven-point scale from 1= "strong disagreement" to 7 = "strong agreement". The scale has demonstrated reliability and validity (Campbell et al., 2004). The Polish version was obtained by translation and independent back-translation and then validated by correlating the scale to the other measures used (Żemojtel-Piotrowska et al., 2015).

The Rosenberg Self-Esteem Scale (Rosenberg, 1965) is a 10-item measure of selfesteem comprising statements related to overall feelings of self-worth and self-acceptance (e.g. "I am able to do things as well as most other people"). Responses were scored on a fourpoint scale ranging from $1=$ "strongly disagree" to $4=$ "strongly agree" in the English sample and from $1=$ "strongly disagree" to $5=$ "strongly agree" in the Polish sample. The scale has demonstrated reliability and validity (Rosenberg, 1965).

\section{Administration of Measures}

In both samples, the participants were volunteers. In Poland the participants were administered paper-and-pencil versions of the questionnaire, while in England the participants completed the studies online. Appropriate ethical approval was obtained from the relevant 
university research ethics committee prior to data collection. The consent form provided before proceeding with the study contained statements regarding the nature of the study, anonymity of the data use, withdrawal from the experiment, and how the data would be stored in a coded form. Participants were rewarded with credit course points.

\section{Correlation Analyses}

\section{Results}

Correlations and reliabilities of all variables are reported in Table 1. They are generally comparable across both samples. As can be noted, the changing of the answering format for the Polish version of the NPI-13 resulted in similar reliability to the English version.

Table 1

Cronbach's alphas of, and Pearson product moment correlations coefficients between, all measures used in the study

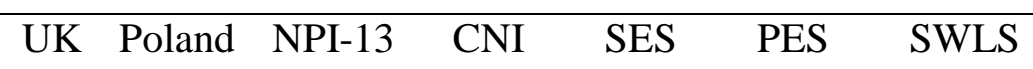

$\alpha \quad \alpha$

Narcissistic Personality

$.70 \quad .74$

$.39 * * *$

$.33 * * *$

$.43^{* * *} .13^{* *}$

Inventory

Communal Narcissism

$.91 \quad 90 \quad .36 * * *$

.12

$.48 * * * \quad-.00$ 
Inventory

$\left(.46^{* * *}\right)$

Self-Esteem Scale

$.90 \quad .87$

$.29 * * *$

$.39 * * *$

$-.02$

$.43 * * *$

$(.35 * * *)$

Psychological Entitlement

$.89 \quad .85$

$.43 * * *$

$.36 * * * \quad .22 * *$

$-.26 * *$

Scale

$$
(.56 * * *)
$$

Satisfaction with Life Scale

$.87 \quad .84 \quad .16^{*}$

$.29 * * * \quad .42 * * * \quad .04$

$* p<.05 ; * * p<.01 ; * * * p<.001$

Note. Due to different response scales for the SWLS in the two samples, row scores were standardized. Correlation coefficients for the UK sample are presented above the diagonal and those for the Polish sample below the diagonal. Correlations for the Polish sample without the recoding of the NPI-13 responses (as described in the method section) are presented in parentheses.

\section{Measurement Model}

We conducted structural equation modelling (SEM) to specify the relationships between the variables. We used confirmatory factor analysis to evaluate the measurement of latent factors. All constructs were measured by parcels, which were aggregates of particular items instead of single items. Each parcel contained from two to four items, depending on which scale was being used. For instance, the SWLS comprised two parcels (one with three items and another with two) and the CNI comprised four. The remaining scales were measured using three parcels. The parcels for the SES were created by balancing the number of negative and positive items within each parcel. The parcels for the NPI were created to reflect the three-facet structure of the scale. As both missing items were from the entitlement subscale, this parcel contained only two items. The item parcels were used to reduce 
estimation errors, improve model fit, and stabilize the parameter estimates (Matsunaga, 2008). All latent factors were measured using at least two parcels and all parcels loaded significantly on a proper factor $(p<.001$, all factor loadings higher than .4$)$.

\section{Structural Model}

The structural model assessed the impact of agentic and communal narcissism on satisfaction with life, with psychological entitlement and self-esteem used as mediators. A summary of the model is shown in Figure 1.

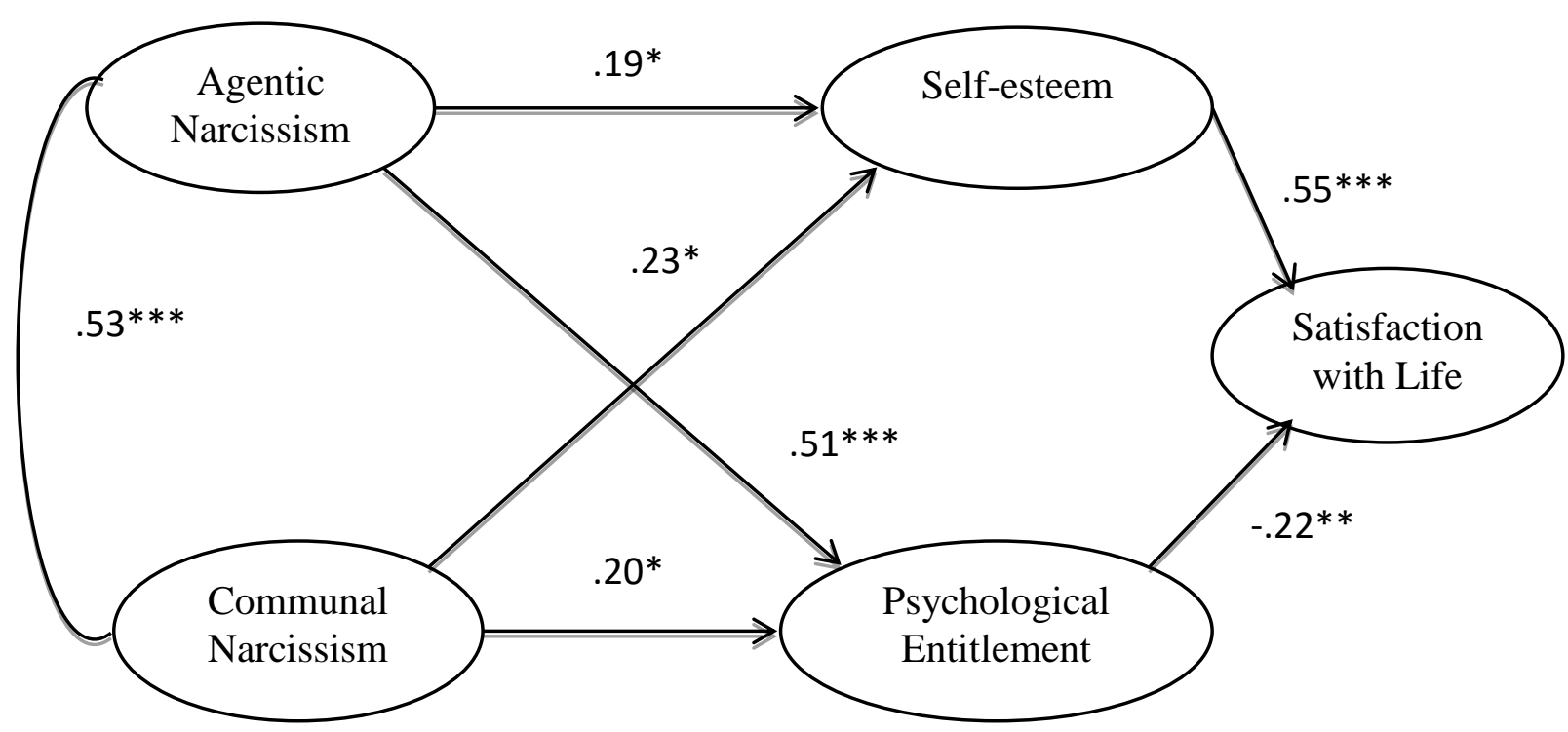

Figure 1. Model of interrelations between agentic and communal narcissism, self-esteem, psychological entitlement, and satisfaction with life

Model comparison indicated that all paths were invariant across the two countries, with the difference $(\Delta$ Tucker Lewis Index $[\mathrm{TLI}]=-.001)$ between the unconstrained model (assuming all model parameters to be different across samples) and the constrained model (assuming equal structural residuals across samples) lower than the recommended cut-off of .01 (Chen, 2007). To assess the goodness of fit of the model we used well-recognized cut-off criteria: the root mean square error of approximation (RMSEA) and standardized root mean square residual (SRMR) were both required to be smaller than .08, and the TLI or nonnormed fit index and the comparative fit index (CFI) were both required to be larger than 90 
(Lance, Butts, \& Michels, 2006). The structural constrained model demonstrated a reasonable fit to the data: $\chi^{2}(188, n=248)=317.08, p<.001, \mathrm{CFI}=.94, \mathrm{TLI}=.93, \mathrm{SRMR}=.08$; RMSEA $=.049(.04-.06), p$ close $=.540$.

All indirect effects tested in the model were significant. The indirect effects of agentic narcissism on life satisfaction were stronger $(\beta=0.16)$ than those of communal narcissism $(\beta$ $=0.07$ ); all direct effects of narcisism on life satisfaction were null, pointing to full mediation in each case. There were no differences in the effects sizes for agentic and communal narcissism in relation to self-esteem. The effect of agentic narcissism on psychological entitlement was stronger than the analogous effect of communal narcissism.

\section{Discussion}

The current study examined the positive (via self-esteem) and negative (via psychological entitlement) associations between grandiose narcissism and satisfaction with life. Congruent with the first assumption about both positive and negative effects of grandiose narcissism on subjective well-being, we found two separate mediational paths explaining the influence of narcissism on satisfaction with life. The first is via self-esteem, which acts as an enhancement to satisfaction with life; the second is via psychological entitlement, which imposes a cost on satisfaction with life. This "mixed blessing" of narcissism (see Paulhus, 1998) could be useful in explaining inconistent findings on the relationship between narcissism and happiness.

Second, congruent with the agentic-communal model (Gebauer et al., 2012), we found parallel results for both forms of narcissism, demonstrating a positive association with selfesteem and psychological entitlement, and similar indirect effects on satisfaction with life. The only difference was that the relationship between agentic narcissism and psychological entitlement was stronger than that between communal narcissism and psychological entitlement. However, this difference could be explained by the structure of NPI-13, which 
includes the subscale of entitlement/exploitativeness. Both the NPI and the PES measure psychological entitlement. Generally, our findings suggest that the costs and benefits of agentic and communal narcissism for subjective well-being are the same.

The most important limitation of the current study is the cross-sectional character of the data. Generally, cross-sectional data do not allow for causal interpretations. However, recent studies support the proposal that narcissism influences subjective well-being, and this relationship is mediated by a positive evaluation in the agentic domain (Zajenkowski \& Czarna, 2015). Therefore, the causal model proposed is not without merit.

The current work provides evidence supporting a model of the relationship between agentic-communal narcissism, self-esteem, psychological entitlement and satisfaction with life. It would be of interest to widen out the consideration of well-being, notably beyond one indicator of subjective well-being to other affective components of subjective well-being or psychological well-being (Keyes, Shmotkin, \& Ryff, 2002). Finally, given that our findings originated from European samples and as there has been some consideration of the differing nature of narcissism in collectivistic societies (Twenge \& Campbell, 2009), it would be interesting to determine the extent to which these models were evident in such cultures.

The findings supplement current knowledge on grandiose narcissism, in terms of both agentic and communal narcissism, showing that psychological entitlement is its important epiphenomenon, as outlined by other scholars (e.g. Freud, 1914; Raskin \& Terry, 1988). While previous research focused on the role of self-esteem in the functioning of narcissistic individuals, the current findings suggest some psychological costs of narcissism, such as unrealistic expectations of others.

\section{References}

Campbell, K.W., Bonacci, A.M., Shelton, J., Exline, J.J., \& Bushman, B.J. (2004). 
Psychological entitlement: Interpersonal consequences and validation of self-report measure. Journal of Personality Assessment, 83, 29-45. DOI:

10.1207/s15327752jpa8301_04

Chen, F.F. (2007). Sensitivity of goodness of fit indexes to lack of measurement invariance. Structural Equation Modeling, 14, 464-504.

Diener, E., Emmons, R.A., Larsen, R.J., \& Griffin, S. (1985). The Satisfaction with Life Scale. Journal of Personality Assessment, 49, 71-75.

Freud, S. (1914). On Narcissism. The Standard Edition of the Complete Psychological Works of Sigmund Freud, Volume XIV (1914-1916): On the History of the Psycho-Analytic Movement, Papers on Metapsychology and Other Works, 67-102.

Gebauer, J. E., Sedikides, C., Verplanken, B., \& Maio, G. R. (2012). Communal narcissism. Journal of Personality and Social Psychology, 103, 854-878. DOI: 10.1037/a0029629

Gentile, B., Miller, J.D., Hoffman, B.J., Reidy, D.E., Zeichner, A., \& Campbell, K.W. (2013). A test of two brief measures of grandiose narcissism: The Narcissistic Personality Inventory-13 and the Narcissistic Personality Inventory-16. Psychological Assessment, 25, 1120-1136. DOI: $10.1037 / \mathrm{a} 0033192$

Keyes, L.M., Shmotkin, D., \& Ryff, C.D. (2002). Optimizing well-being: The empirical encounter of two traditions. Journal of Personality and Social Psychology, 8, 10071022. DOI: $10.1037 / / 0022-3514.82 .6 .1007$

Lance, C.E., Butts, M.M., \& Michels, L.C. (2006). The sources of four commonly reported cut-off criteria: What did they really say? Organizational Research Methods, 9, 202220. DOI: $10.1177 / 1094428105284919$

Matsunaga, M. (2008). Item parceling in structural equation modeling: A primer. Communication Methods and Measures, 2, 260-293. DOI:10.1080/19312450802458935 
Miller, J.D., \& Campbell, K.W. (2008). Comparing clinical and social-personality approaches to narcissism. Journal of Personality, 76, 449-476. DOI: 0.1111/j.14676494.2008.00492.x

Miller, J.D., Hoffman, B.J., Gaughan, E.T., Gentile, B., Maples, J., \& Campbell, W.K. (2011). Grandiose and vulnerable narcissism: A nomological network analysis. Journal of Personality, 79, 1013-1042.

Paulhus, D.L. (1998). Interpersonal and intrapsychic adaptiveness of trait self-enhancement: A mixed blessing? Journal of Personality and Social Psychology, 74, 11971208. http://dx.doi.org/10.1037/0022-3514.74.5.1197

Raskin, R., \& Terry, H. (1988). A principal-components analysis of the Narcissistic Personality Inventory and further evidence of its construct validity. Journal of Personality and Social Psychology, 54, 890-902.

Rose, P. (2002). The happy and unhappy faces of narcissism. Personality and Individual Differences, 33, 379-391. DOI: 10.1016/S0191-8869(01)00162-3

Rosenberg, M. (1965). Society and the Adolescent Self-image. Princeton, NJ: Princeton University Press.

Sedikides, C., Rudich, E.A., Gregg, A.P., Kumashiro, M., \& Rusbult, C. (2004). Are normal narcissists psychologically healthy? Self-esteem matters. Journal of Personality and Social Psychology, 87, 400-416. DOI: 10.1037/0022-3514.87.3.400

Twenge, J., \& Campbell, W.K. (2009). The Narcissism Epidemic. Life in the Age of Entitlement. London: Free Press. 
Zajenkowski, M., \& Czarna, A. (2015). What makes narcissists unhappy? Subjectively assessed intelligence moderates the relationship between narcissism and psychological well-being. Personality and Individual Differences, 77, 50-54. DOI:

10.1016/j.paid.2014.12.045

Żemojtel-Piotrowska, M., Clinton, A., \& Piotrowski, J. (2014). Agentic and communal narcissism and subjective well-being: Are narcissistic individuals unhappy? A research report. Current Issues in Personality Psychology, 2, 8-16. DOI:

$10.5114 / \operatorname{cipp} .2014 .430$

Żemojtel-Piotrowska, M. Piotrowski, J., Cieciuch, J., Calogero, R., Van Hiel, A., (...), \& Wills-Herrera, E. (2015). Measurement of psychological entitlement in 28 countries. European Journal of Psychological Assessment, in press. 
NARCISSISM, SATISFACTION WITH LIFE AND ENTITLEMENT 